\title{
Interactive comment on "APIFLAME v2.0 trace gas and aerosol emissions from biomass burning: application to Portugal during the summer of 2016 and evaluation against satellite observations of CO (IASI) and AOD (MODIS)" by Solène Turquety et al.
}

Anonymous Referee \#2

Received and published: 2 December 2019

This study presents an updated version of the APIFLAME model to estimate biomass burning emissions. The new biomass burning (BB) emissions dataset was evaluated by using the offline air quality model CHIMERE for summer 2016, focusing on the intensive wildfires in Portugal. The major update in APIFLAME is merging the burned area and fire radiative power datasets in order to simulate the temporal variability of the BB emissions and also incorporate the small fires. Various configurations of the APIFLAME model are presented here to show the range of the uncertainties in the BB

Printer-friendly version

Discussion paper 
emission estimates.

Development of accurate and fine-scale BB inventories is crucial for assessing the impact of fires on air quality and climate. The methods used in the study are novel. However, the paper requires major improvement for publication in GMD.

First, some of the wording in the text has to be improved. Some sentences aren't clear.

Other major comments:

I suggest changing the title of the paper to state that this study isn't only evaluating the BB emissions, but also the CHIMERE model. I know in a number of studies BB emissions were evaluated by using the atmospheric models. However, this gives a false impression that different BB emissions can be evaluated accurately by plugging them into the air quality models. As the authors note, there are many uncertainties in the modeling of the plume injection height, tracer transport and mixing, and atmospheric chemistry in the air quality and atmospheric chemistry models. These uncertainties have profound effect on the performance of the atmospheric models. This point has to be made clear in the Abstract as well.

It has to be emphasized that the APIFLAME2.0 can be used for the retrospective studies, not for forecasting. There are significant challenges in forecasting BB emissions, especially on the regional scales. Sometime it's assumed that using the new satellite data the forecasting of BB emissions can be easily done. The satellite FRP data provide information about the state of a fire intensity, unless the satellite scans are obscured by dense smoke or cloudiness. It's still very hard to forecast the spatial and temporal variability of the BB emissions for next hours and days.

The burned area data is the primary source of the information to estimate the BB emissions in APIFLAME. I suggest adding a short description of the burned area dataset and associated uncertainties.

Printer-friendly version

In section 4.1 it's stated that high FRP points correspond to larger burned areas. While

Discussion paper 
this assumption is true in general, sometimes the MODIS instruments report very high FRPs ( 1000MW) for small agriculture fires, for example when the overpass time coincides with the intensive flaming phase.

One of the key uncertainties in modeling of the BB emissions is the estimating flaming and smoldering emissions. This topic isn't discussed in the paper. What emission factors (EFs) did the authors use to estimate the emissions of $\mathrm{CO}$ and other chemical species? For smoldering or flaming phases?

When the plume rise parameterization is used, how the emissions are partitioned in CHIMERE? What vertical distribution was used for the BB emissions in the model with Sofiev et al. plume rise parameterization?

L.405: The studies show that as the semi-volatile POA species emitted by fires evaporate partially, more SOA forms downwind of fires. Overall these two processes compensate each other. How these processes are parameterized in CHIMERE?

How the AOD is calculated from the CHIMERE model output? In the literature the reported aerosol extinction coefficients for smoke vary from one study to another. Was any hygroscopicity assumed for the modeled BB aerosols?

Some text and figures can be moved to SI, e.g. Figure 3, Tables A1-2.

Using the MISR data to constrain the injection heights in the model is a reasonable approach. However, the MISR typically misses the most intensive stages of the fire evolution (occurring during the afternoon hours). This will lead to underestimate of the fire injection heights overall.

Minor comments:

L. 540: Move "The ability..." to the Introduction.

Printer-friendly version

Discussion paper

Interactive comment on Geosci. Model Dev. Discuss., https://doi.org/10.5194/gmd-2019-210, 2019. 